\title{
A conformance testing for communication protocols modeled as a set of DFSMs with common inputs
}

Atsushi Fukada ${ }^{\dagger}$, Tadashi Kaji $i^{\dagger *}$, Teruo Higashino ${ }^{\dagger}$, Kenichi Taniguchi ${ }^{\dagger}$ and Masaaki Mori ${ }^{\dagger \dagger}$

$\dagger$ Dept. Information and Computer Sciences,

Osaka University, Toyonaka, Osaka 560, Japan

Tel : +81-6-850-6607 Fax : +81-6-850-6609

E-mail : \{a-fukada, higashino, taniguchi\}@ics.es.osaka-u.ac.jp

*Currently Hitachi Co. Ltd. E-mail : t-kaji@sdl.hitachi.co.jp

††Dept. of Information Processing and Management,

Shiga University, Hikone, Shiga 522, Japan

E-mail : mori@biwako.shiga-u.ac.jp

\begin{abstract}
In this paper, we propose an effective conformance testing method for a subclass of protocols modeled as a set of DFSMs. The number of test cases in the proposed method is only proportional to the sum of those of states and transitions in a given set of DFSMs. In our method, we find a characterization set for each DFSM, which is used to test the DFSM alone in W $\dot{\mathrm{p}}$-method, and the union of the characterization sets is used as a characterization set for the total system. For a set of DFSMs with common inputs, there may exist two or more tuples of states that have correct responses against a given characterization set. So, in order to identify each state $s$ in a DFSM, we find a characterization set with some specific properties. Then we select a suitable
\end{abstract}


tuple of states containing the state $s$, and identify the state $s$ by checking their response to the characterization set.

\section{Keywords}

Verification, protocol testing, test case selection and test coverage

\section{INTRODUCTION}

Conformance testing for communication protocols is highly effective to develop reliable communication systems. There are many research efforts on generating conformance test cases mechanically, which are well known as the TTmethod [7], W-method [1], DS-method [2], UIO-method [9], and so on. Furthermore, there are some research papers focusing on more effective methods for generating test sequences. These efforts were mainly made for communication protocols modeled as a single deterministic finite state machine (DFSM). Recently similar research efforts have been done on non-deterministic or concurrent models $[4,5,6,11,12]$.

According to the progress of computer networks, many kinds of protocols, which use several channels in parallel, are proposed. For such a protocol, it is quite natural that a protocol with several channels is considered as a set of DFSMs, each of which controls one channel and competes with other DFSMs for taking common inputs. Since a common input is taken by some DFSMs, the whole behavior of a given set of DFSMs is non-deterministic.

As a conformance testing method for such a non-deterministic FSM (NFSM) model, there is the GWp-method [6]. Such a method can be applied to a set of DFSMs mentioned above. However, since, in general, all reachable tuples of states of the DFSMs are considered as the states of the total system, the number of states of the total system is proportional to the product of those of the DFSMs. So, the number of generated test cases is also proportional to the product of those of the DFSMs. One of the methods free from such a drawback is to carry out the conformance testing for each DFSM independently. For example, the testing method in [5] is based on this idea and it treats protocols with communications among FSMs and internal actions. Since this method is based on the TT-method, it cannot identify the state after each transition is executed.

In this paper, for a specification modeled as a set of DFSMs with common inputs, we assume that the implementation under test (IUT, for short) is also modeled as a set of DFSMs (sub-IUTs) where the number of states of each sub-IUT does not exceed that of the corresponding DFSM in the specification. Under this assumption, we propose a testing method based on the GWpmethod where the number of test cases is only proportional to the sum of those of states and transitions in a set of DFSMs. It identifies all states of DFSMs and confirms all transitions even if the IUT has any number of faults. 
The proposed method uses the union of characterization sets $W_{i}$ for all DFSMs $A_{i}$ as a characterization set for the total system. Here, we assume that we can generate a characterization set which can identify all states in each DFSM even if the common inputs in the characterization set are received by several DFSMs non-deterministically. In order to identify a state $s$ of a DFSM, we select a suitable tuple of states containing the state $s$, and identify the state $s$ by checking its response to the characterization set.

The paper is structured as follows. In Section 2, we explain the model used in this paper, and then we define its fault model. In Section 3, the outline of the GWp-method for testing NFSMs is explained. In Section 4, we propose a testing method. In Section 5, the correctness of the proposed testing method is described. An example is given in Section 6. We conclude the paper in Section 7.

\section{A SET OF DFSMS WITH COMMON INPUTS}

\subsection{Specification and Its Implementation}

Definition 1 (Finite State Machine) A finite state machine (FSM) is defined as the following 6-tuple,

$$
A=\left(S, X, Y, \delta, \lambda, s_{0}\right)
$$

Here, $S, X$ and $Y$ are a finite set of states, a finite set of inputs and a finite set of outputs, respectively. $\delta$ is a transition function $(S \times X \rightarrow S)$, and $\lambda$ is an output function $(S \times X \rightarrow Y) . s_{0}$ is the initial state of $A$.

For two states $s$ and $t$, we say that $s$ is equivalent to $t$ if $\lambda\left(s, \sigma^{i}\right)=\lambda\left(t, \sigma^{i}\right)$ holds for any input sequence $\sigma^{i}$. We say that a FSM $M_{1}$ is equivalent to a FSM $M_{2}$ if the initial state of $M_{1}$ is equivalent to that of $M_{2}$. A FSM $M$ is said to be minimal if, for any two different states $s$ and $t$ of $M, s$ is not equivalent to $t$. We say that a FSM $M$ is completely specified (or complete) if both transition function and output function are defined for any pair of a state and an input. In this paper, if a FSM is not completely specified, we make the FSM complete as follows : For each pair of a state $s$ and an input $x$ whose transition and output functions are undefined, we add a new transition from $s$ to itself whose output is empty and make the FSM complete. Here, we denote such an empty output by " $\varepsilon$ ". For such a new transition $x / \varepsilon$ from a state $s$, we say that the FSM ignores input $x$ at state $s$.

A FSM is said to be initially connected if there exists a transition sequence from the initial state to any state of the FSM, where the transition sequence may be a null sequence.

A FSM is said to be deterministic if, for any pair of a state $s$ and an input $x$, $\delta(s, x)$ and $\lambda(s, x)$ are uniquely defined. Such a FSM is called a deterministic 
FSM (DFSM). A FSM that is not a DFSM is called a non-deterministic FSM (NFSM). If two non-deterministic transitions from a state have the same outputs $b$ for an input $a$, then we say that the non-deterministic transitions $(a / b$ and $a / b)$ are non-observable. Otherwise (for example, $a / b$ and $a / c$ ), we say that they are observable non-deterministic transitions. We say that a NFSM is observable NFSM (ONFSM) if all the non-deterministic transitions in the NFSM are observable [10].

Next, we define a set of DFSMs used in this paper. We call this model as Coupled DFSMs.

Definition 2 (Coupled DFSMs) Coupled DFSMs are a $k$-tuple,

$$
A=\left(A_{1}, A_{2}, \ldots A_{k}\right)
$$

where $A_{1}, A_{2}, \ldots, A_{k}$ are DFSMs, respectively. Also, each $A_{i}(1 \leq i \leq k)$

$$
A_{i}=\left(S_{i}, X_{i}, Y_{i}, \delta_{i}, \lambda_{i}, s_{i 0}\right)
$$

must be a complete, initially connected and minimal DFSM. Furthermore, we suppose there is a reset operation so that the whole Coupled DFSMs are reset to their initial states at a time.

Here, an input such as $x \in X_{i} \cap X_{j}$ is called a common input. If a common input $x$ is given to Coupled DFSMs from the external environment, one of DFSMs takes the input $x$ non-deterministically and the chosen DFSM returns a response (output). Here, it is assumed that $A_{i}$ is not chosen whenever $A_{i}$ ignores the input $x$ and $A_{j}$ does not.

Definition 3 (Specifications of Communication Protocols) A specification of a communication protocol dealt with in this paper is given as Coupled DFSMs

$$
A=\left(A_{1}, A_{2}, \ldots A_{k}\right)
$$

consisting of $k$ DFSMs. We also suppose that this specification does not contain any non-observable and non-deterministic transitions as a whole.

$A_{i}(1 \leq i \leq k)$ is said to be a sub-specification of $A$. Since we assume that a specification does not contain any non-observable and non-deterministic transitions, if there is a transition $a / b$ in $A_{i}$, there may exist a transition $a / c$ in $A_{j}$. However, there does not have to exist the same transition $a / b$ in $A_{j}$.

Each implementation under test (IUT) $I$ is given as follows.

Definition 4 (Implementation Under Test (IUT)) An implementation under test (IUT) $I$ of a communication protocol is given as Coupled DFSMs consisting of $k$ DFSMs

$$
I=\left(I_{1}, I_{2}, \ldots, I_{k}\right)
$$

where each $I_{j}$ must satisfy the following properties: $I_{j}$ has the same set $X_{j}$ 


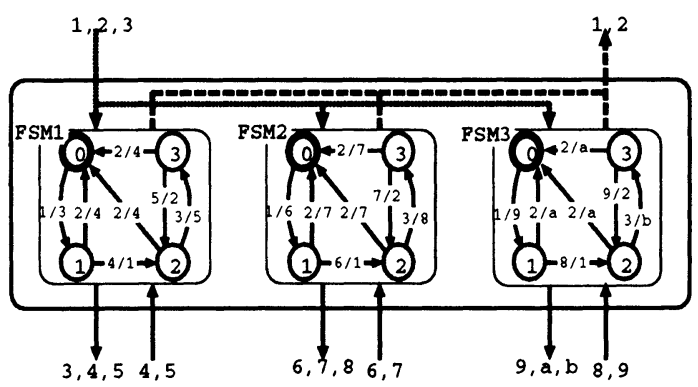

Figure 1 Example of Multi-Link Protocol.

Table 1 Inputs

\begin{tabular}{cl}
\hline & Inputs \\
\hline 1 & link inc. req. \\
2 & link dec. req. \\
3 & data trans. req. \\
$4,6,8$ & connect confirm \\
$5,7,9$ & data trans. ack \\
\hline
\end{tabular}

Table 2 Outputs

\begin{tabular}{cl}
\hline & Outputs \\
\hline 1 & connect confirm \\
2 & data trans. ack \\
$3,6,9$ & link inc. indication \\
$4,7, \mathrm{a}$ & link dec. indication \\
$5,8, \mathrm{~b}$ & data trans. indication \\
\hline
\end{tabular}

Table 3 States

\begin{tabular}{ll}
\hline & \multicolumn{1}{c}{ States } \\
\hline 0 & disconnected \\
1 & wait(connection) \\
2 & connected \\
3 & wait(data) \\
\hline
\end{tabular}

of input symbols as $A_{j}$. A set of output symbols in $I_{j}$ is equal to the set $Y=Y_{1} \cup Y_{2} \cup \ldots \cup Y_{k}$ that is the set of all output symbols used in the protocol. That is, there may exist a fault that $I_{j}$ gives an output $y$ such as $y \notin Y_{j} \wedge y \in Y_{i}(i \neq j)$. The number of states of each $I_{j}$ does not exceed that $\left(\left|S_{j}\right|\right)$ of $A_{j}$.

We also suppose that there exists a reliable reset operation so that the whole Coupled DFSMs can be reset to their initial states at a time.

$I_{j}(1 \leq j \leq k)$ is said to be a sub-IUT of $I$. As stated above, we suppose that both a specification $A$ and an IUT $I$ are modeled by the same number $k$ of DFSMs, however, the internal states of $I$ are not observable. That is, the IUT $I$ is considered to be a black-box and will be tested. On the other hand, the specification $A$ must be ONFSM as a whole, but the IUT $I$ could have non-observable and non-deterministic transitions.

Definition 5 (Conformance of Communication Protocol) For a specification $A=\left(A_{1}, A_{2}, \ldots, A_{k}\right)$ and an IUT $I=\left(I_{1}, I_{2}, \ldots, I_{k}\right)$ of communication protocols stated above, we define that $I$ is a correct implementation of $A$ if each sub-IUT $I_{j}$ of $I$ is equivalent to the corresponding sub-specification $A_{j}$ of $A$. 


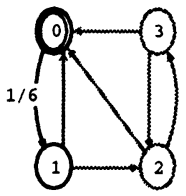

FSM1

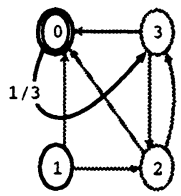

FSM2

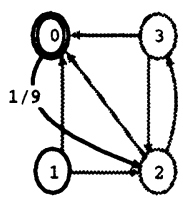

FSM3

Figure 2 A Faulty IUT for Fig. 1.

\subsection{Example Protocol in Our Model}

We consider the protocol shown in Fig. 1 as an example. Fig. 1 represents a specification of a protocol which can dynamically vary the number of links between the lower and upper layers. On this specification, at most three links are set up by the orders from the upper layer, where the finite state control for each link is modeled as a DFSM. Table 1,2,3 represents the contents of inputs, outputs and states.

Whenever a "Link Increase request" is issued to this protocol by the upper layer, a link is newly set up by one of DFSMs which has not set up any link to the lower layer. In the case of "Link Decrease request" by the upper layer, one of DFSMs which has been holding a link to the lower layer cuts the link. Only executable DFSMs can compete with each other for these Link Increase/Decrease requests. That is, when an input 1 ("Link Increase request") is issued, one of DFSMs with states where the input 1 is not ignored is chosen by non-deterministically and responds to the input 1 . For example, in case that FSM2 is in the state where the input 1 is ignored (such as state 1 in Fig. 1), FSM2 is never chosen. If all DFSMs are in the states where the input 1 is ignored, we consider that one of them ignores the input 1 . In this specification, we can easily check which DFSM responds to each Link Increase/Decrease request from its output. That is, the specification is modeled as an ONFSM as a whole. Fig. 2 is an example of a faulty implementation of the protocol shown in Fig. 1. Hereafter, we assume that the italic-faced FSM $i$ denotes a sub-IUT corresponding to the sub-specification FSMi. At the tuple of states $(0,0,0)$ of the Coupled DFSMs, their faults cannot be detected easily, since as a whole the outputs for the input 1 are the same as the specification (the set of 3,6 and 9).

\section{OUTLINE OF GWP-METHOD}

The GWp-method[6] is an extended version of the Wp-method so that ONFSMs can be dealt with. In the GWp-method, testing consists of two parts : one is state identification whether there exist all states of the specification in a given IUT, and the other is confirmation of transitions whether all tran- 
sitions of the specification are correctly implemented in a given IUT. Both of testing are carried out by checking whether the output sequences obtained by applying test sequences to the IUT are equal to those from the specification. Since an ONFSM has non-deterministic actions, in general, a number of variety of output sequences may be produced as the response for an input sequence. So, in the GWp-method, we give each test sequence to the IUT for several times. If the obtained set of the output sequences is not equal to that from the specification, we regard that the IUT has a fault. If the sets are the same, we regard that the IUT returns the correct response for the test sequence and continue the test using other test sequences. If the IUT returns the correct response for all test sequences, then we regard that the IUT is a correct implementation of a given specification.

The set of test sequences in the GWp-methods is given by constructing two kinds of sets of sequences : the characterization set (W set) and state cover (V set).

Definition 6 (Characterization Set) The characterization set W for a FSM is given as a set of input sequences called characterization sequences. Each state in a given FSM must be uniquely identified by observing the set of output sequences obtained by applying all input sequences in $\mathrm{W}$.

\section{Definition 7 (Transfer Sequences to Goal States) A transfer sequence} to a state $s$ in a FSM is an input sequence which makes the FSM move from the initial state to the goal state $s$. The set $\mathrm{V}$ of transfer sequences to all states in the FSM is called a state cover.

In NFSM models, there may exist several reachable states for a given transfer sequence because of non-deterministic behavior. Therefore, for an ONFSM, if the ONFSM does not produce the expected output sequence for a given transfer sequence, we decide that the ONFSM does not reach the expected state because of non-deterministic behavior, and try the test again.

Definition 8 (Test Suite) The test suite for state identification is the concatenation $V . W$ of $\mathrm{V}$ and $\mathrm{W}$. The test suite for confirmation of transitions is defined as follows using $\mathrm{V}, \mathrm{W}$ and $\mathrm{X}$ (the set of input symbols) $: V . X \oplus W=$ $\left\{\sigma . w \mid \sigma \in V . X, s_{0} \stackrel{\sigma}{\rightarrow} s_{i}, w \in W\right\}$

\section{PROPOSED TESTING METHOD}

In the proposed method, for a specification $A=\left(A_{1}, A_{2}, \ldots, A_{k}\right)$ and an implementation $I=\left(I_{1}, I_{2}, \ldots, I_{k}\right)$ modeled as Coupled DFSMs, first, we give some conditions for the characterization set used in the testing. Then, using the characterization set satisfying the conditions, we will carry out the test 
whose cost is proportional to the sum of the numbers of states and transitions in the DFSMs. Like the GWp-method, the proposed testing method is divided into the following two parts: (1) state identification and (2) confirmation of transitions.

\subsection{Construction of Characterization Set}

In the proposed method, for state identification, we assume that we can construct a characterization set $W_{i}$ for each sub-specification $A_{i}$ which satisfies the following two conditions(Def.9 and 10).

Definition 9 (Condition for common inputs) For each characterization set $W_{i}$ containing common inputs, $W_{i}$ must be able to identify each state in $A_{i}$ even if $A_{i}$ ignores the common inputs in $W_{i}$.

Then, we construct the following set $W$.

$$
W=W_{1} \cup W_{2} \cup \cdots \cup W_{k}
$$

For each input sequence $\sigma$ in $W$, let $\overline{\sigma_{i}}$ denote the input sequence obtained from $\sigma$ by deleting all the input symbols which the sub-specification $A_{i}$ cannot respond to. And let $W_{i}^{\prime}$ denote the set of $\overline{\sigma_{i}}$ for all input sequences $\sigma$ in $W$. Here, we also treat each $W_{i}^{\prime}$ as a characterization set for $A_{i}$.

As an example, let's consider the protocol in Fig. 1. Suppose that the following characterization sets are constructed.

$$
W_{1}=\{35,4,5\}, W_{2}=\{16,7,6\}, W_{3}=\{18,9,8\}
$$

Then, we obtain the characterization set $W$ for the total system as follows:

$$
W=\{35,4,5,16,7,6,18,9,8\}
$$

Also, the characterization sets

$$
W_{1}^{\prime}=\{35,4,5,1\}, W_{2}^{\prime}=\{16,7,6,3\}, W_{3}^{\prime}=\{18,9,8,3\}
$$

are obtained.

Here, we must take the above condition into consideration for the construction of $W_{1}$. In Table 4, even if we make a smaller characterization set $W_{1}^{\prime \prime}=\{35,4\}$, it can identify four states of FSM1. But the common input 3 in $W_{1}^{\prime \prime}$ may be taken by one of other DFSMs, that is, in Table 4, the corresponding output 5 with underline may be changed to $\varepsilon$. In this case, we cannot distinguish state 2 from state 3 . So it is necessary to construct the characterization set $W_{1}$ which can identify each state in FSM1 even for such a case. That is the reason why we add another sequence 5 to $W_{1}$. By adding the sequence 5 to $W_{1}$, we can distinguish state 2 from state 3 even if the common input 3 is taken by another DFSM. Also in Tables 5 and 6, we add sequences 6 and 8 to $W_{2}$ and $W_{3}$, respectively, from the same reason. 
Table $4 W_{1}$

\begin{tabular}{cccc}
\hline State & 35 & 4 & 5 \\
\hline 0 & $\varepsilon \varepsilon$ & $\varepsilon$ & $\varepsilon$ \\
1 & $\varepsilon \varepsilon$ & 1 & $\varepsilon$ \\
2 & $\underline{5} 2$ & $\varepsilon$ & $\varepsilon$ \\
3 & $\varepsilon 2$ & $\varepsilon$ & 2 \\
\hline
\end{tabular}

Table $5 \quad W_{2}$

\begin{tabular}{cccc}
\hline State & 16 & 7 & 6 \\
\hline 0 & $\underline{6} 1$ & $\varepsilon$ & $\varepsilon$ \\
1 & $\varepsilon 1$ & $\varepsilon$ & 1 \\
2 & $\varepsilon \varepsilon$ & $\varepsilon$ & $\varepsilon$ \\
3 & $\varepsilon \varepsilon$ & 2 & $\varepsilon$ \\
\hline
\end{tabular}

Table $6 W_{3}$

\begin{tabular}{cccc}
\hline State & 18 & 9 & 8 \\
\hline 0 & $\underline{91}$ & $\varepsilon$ & $\varepsilon$ \\
1 & $\varepsilon 1$ & $\varepsilon$ & 1 \\
2 & $\varepsilon \varepsilon$ & $\varepsilon$ & $\varepsilon$ \\
3 & $\varepsilon \varepsilon$ & 2 & $\varepsilon$ \\
\hline
\end{tabular}

Definition 10 (Condition for response of other DFSMs) Let $C_{i}$ denote the set of common inputs contained in $W_{i}^{\prime}$. We assume that we can construct each $W_{i}^{\prime}$ such that every $A_{j}(j \neq i)$ has a state which ignores all common inputs in $C_{i}$.

For state identification, we only assume the above two conditions. However, for confirmation of transitions, we need a further assumption. If a state $s$ in a sub-specification $A_{i}$ ignores a common input $x$, and if another subspecification $A_{j}$ produces an output $y$ at every state for the common input $x$. In this case, we cannot confirm logically that $s \stackrel{x / \varepsilon}{\rightarrow} s$ in $A_{i}$ is correctly implemented. The IUT produces the same outputs as the total system even if $I_{i}$ produces the output $y$ for the input $x$ at state $s$, since at any tuple of states containing state $s, I_{j}$ produces the output $y$.

Therefore, we also give the following assumption for each sub-specification $A_{i}$.

Definition 11 (Assumption for each DFSMs) Let $D$ denote the set of common inputs. We assume that every $A_{i}$ has a state which ignores each common input in $D$.

In general, even if there exists a characterization set for each sub-specification, there may not exist a characterization set which satisfies the above three conditions. However, for most cases that there are not so many common input symbols in each characterization set, we believe that we can construct characterization sets which satisfy the above three conditions (for example, see an example in Section 6).

\subsection{State Identification}

We identify all the states in DFSMs as follows.

- Selection of tuples of states

Let $s_{j}^{i}$ denote a state in $A_{j}(j \neq i)$ which can ignore all inputs in $C_{i}$. For each state $s_{p}$ of $A_{i}$, we treat $s s_{p}^{i}=\left(s_{1}^{i}, s_{2}^{i}, \cdots, s_{k}^{i}\right)\left(s_{i}^{i}=s_{p}\right)$ as the tuple of 
states to identify the state $s_{p}$. Note that when we give any input in $C_{i}$ to the tuple of states $s s_{p}^{i}$, only the state $s_{p}$ of $A_{i}$ can make a response.

- Giving test sequences

We give a test suite $V \cdot W_{i}^{\prime}$ to the tuples of states constructed above for several times. Here, each $v_{p}^{i}$ in $V$ is the sequence obtained by concatenating all transfer sequences $v_{1}^{i}, v_{2}^{i}, \cdots, v_{k}^{i}$ for the states $s_{1}^{i}, s_{2}^{i}, \cdots, s_{k}^{i}$ in $s s_{p}^{i}$. And we use the same $v_{p}^{i}$ repeatedly while testing for a state. If we get an unexpected output sequence as the result for the transfer sequence $v_{p}^{i}$, we consider that non-deterministic behavior of Coupled DFSMs makes the IUT move to a tuple of states except $s s_{p}^{i}$ and we try to give the transfer sequence $v_{p}^{i}$ again. If we get the correct output sequence for $v_{p}^{i}$, then we observe a response of $s s_{p}^{i}$ for each input of $W_{i}^{\prime}$. If the obtained set of output sequences is not equal to that from the specification, we conclude that the IUT is faulty.

We apply the above method to all states in each sub-specification $A_{i}(1 \leq i$ $\leq k$ ). If we cannot find faulty states, we conclude that we have identified all the states in the IUT.

As an example, we try to identify states of FSM1 in Fig. 1.

Selection of tuples of states

The characterization set $W_{1}^{\prime}$ has common inputs $C_{1}=\{1,3\}$. For example, both state 1 of FSM2 and state 1 of FSM3 ignore these common inputs 1 and 3 . So, we select a tuple of states $(0,1,1)$ for identifying state 0 of FSM1. We select $(1,1,1)$ for state $1,(2,1,1)$ for state 2 and $(3,1,1)$ for state 3 , respectively.

- Giving test sequences

We give the test suite $V . W_{1}^{\prime}$ to the IUT for several times and observe a response from the IUT. This $V$ is a set of transfer sequences to the chosen four tuples, for example, $V=\{11,111,1411,14511\}$. For testing a tuples of states $(2,1,1)$, if the output sequence for a transfer sequence 1411 is not 3169 , we decide that we couldn't transfer the IUT to a tuple of states $(2,1,1)$ because of non-deterministic behavior. Then we try the test again.

\subsection{Confirmation of Transitions}

For a transition $s_{p} \stackrel{x / y}{\rightarrow} s_{q}(x \in X, y \in Y \cup\{\varepsilon\})$ in each sub-specification $A_{i}$, we confirm the transition by dividing the following two cases.

If the input $x$ is not a common input, we give a test suite $v \cdot x . W_{i}^{\prime}$ where $v$ is the same transfer sequence used to identify the state $s_{p}$.

If the input $x$ is a common input, we give a test suite $v . W \cup v \cdot x . W_{i}^{\prime}$. Here, the transfer sequence $v$ in this test suite is constructed as follows. We find a tuple of states $s s_{p}^{i}=\left(s_{1}^{i}, s_{2}^{i}, \cdots, s_{k}^{i}\right)\left(s_{i}^{i}=s_{p}\right)$ where each state $s_{j}^{i}$ of $A_{j}(j \neq i)$ 
ignores the common input $x$. Then, $v$ may be any transfer sequence to $s s_{p}^{i}$. Here, the test suite $v . W$ is used to identify all states in the tuple of states $s s_{p}^{i}$ (note that $W$ is the union of all characterization sets $W_{i}^{\prime}$ for identifying each state $s_{j}^{i}$ ). The test suite $v \cdot x . W_{i}^{\prime}$ is used for confirming the transition and identifying the state which the sub-IUT $I_{i}$ reaches after the transition is executed.

We apply the above method to all transitions. If the IUT is not faulty, we conclude that the implementation of transitions for the specification is correct.

Note that the numbers of test sequences used for identifying states and confirming transitions are proportional to the sum of those of states and transitions in DFSMs, respectively.

\section{CORRECTNESS OF TESTING METHOD}

\subsection{Correctness of State Identification}

For a specification modeled as Coupled DFSMs, we must take the following two notices. One is the existence of the common inputs. Assume that for an identification of a state $s$ in $A_{i}$, we can observe the expected output 1 after providing an input $x$. At this time, the IUT may be implemented badly, since it is possible that $A_{i}$ doesn't give the output 1 and the other $A_{j}$ gives the output. This is the reason why we cannot identify a state even if we can observe the expected response for the IUT. The other is our assumption that the IUT may have non-observable and non-deterministic transitions. On this case, for a given transfer sequence, even if we can observe the expected output sequence, we cannot guarantee that the IUT is led to the tuple of states which we want to lead. The IUT may be led to several tuples of states.

For example, the response of a tuple of states $(2,1,1)$ for $W_{1}^{\prime}$ is,

$$
35 / 5 \varepsilon, 4 / \varepsilon, 5 / \varepsilon, 1 / \varepsilon
$$

Here, a sub-IUT FSM1 may ignore the common inputs 1 and 3 . Then the sub-IUT FSM1 has at least one of states which return the response like the following (there may exist both states) :

$$
(35 / 5 \varepsilon, 4 / \varepsilon, 5 / \varepsilon, 1 / \varepsilon),(35 / \varepsilon \varepsilon, 4 / \varepsilon, 5 / \varepsilon, 1 / \varepsilon)
$$

In order to consider all possibilities stated above, we introduce the state variables in Table 7 where the value of each variable is true if and only if there exists the corresponding state in the sub-IUT FSM1. The above condition can be expressed as the following logical formulas.

- For the tuple of states $(0,1,1), \varphi 11 \vee \varphi 12$

- For the tuple of states $(1,1,1), \varphi 13$

- For the tuple of states $(2,1,1), \varphi 14 \vee \varphi 15$ 


\begin{tabular}{|c|c|c|c|c|c|}
\hline Var & Response for $W_{1}^{\prime}$ & Var & Response for $W_{2}^{\prime}$ & Var & Response for $W_{3}^{\prime}$ \\
\hline$\varphi 11$ & $35 / \varepsilon \varepsilon, 4 / \varepsilon, 5 / \varepsilon, 1 / 3$ & $\varphi 21$ & $16 / 61,7 / \varepsilon, 6 / \varepsilon, 3 / \varepsilon$ & $\varphi 31$ & $18 / 91,9 / \varepsilon, 8 / \varepsilon, 3 / \varepsilon$ \\
\hline$\varphi 12$ & $35 / \varepsilon \varepsilon, 4 / \varepsilon, 5 / \varepsilon, 1 / \varepsilon$ & $\varphi 22$ & $16 / \varepsilon 1,7 / \varepsilon, 6 / \varepsilon, 3 / \varepsilon$ & $\varphi 32$ & $18 / \varepsilon 1,9 / \varepsilon, 8 / \varepsilon, 3 / \varepsilon$ \\
\hline$\varphi 13$ & $35 / \varepsilon \varepsilon, 4 / 1,5 / \varepsilon, 1 / \varepsilon$ & $\varphi 23$ & $16 / \varepsilon 1,7 / \varepsilon, 6 / 1,3 / \varepsilon$ & $\varphi 33$ & $18 / \varepsilon 1,9 / \varepsilon, 8 / 1,3 / \varepsilon$ \\
\hline$\varphi 14$ & $35 / 52,4 / \varepsilon, 5 / \varepsilon, 1 / \varepsilon$ & $\varphi 24$ & $16 / \varepsilon \varepsilon, 7 / \varepsilon, 6 / \varepsilon, 3 / 8$ & $\varphi 34$ & $18 / \varepsilon \varepsilon, 9 / \varepsilon, 8 / \varepsilon, 3 / b$ \\
\hline$\varphi 15$ & $35 / \varepsilon 2,4 / \varepsilon, 5 / \varepsilon, 1 / \varepsilon$ & $\varphi 25$ & $16 / \varepsilon \varepsilon, 7 / \varepsilon, 6 / \varepsilon, 3 / \varepsilon$ & $\varphi 35$ & $18 / \varepsilon \varepsilon, 9 / \varepsilon, 8 / \varepsilon, 3 / \varepsilon$ \\
\hline$\varphi 16$ & $35 / \varepsilon 2,4 / \varepsilon, 5 / 2,1 / \varepsilon$ & $\varphi 26$ & $16 / \varepsilon \varepsilon, 7 / 2,6 / \varepsilon, 3 / \varepsilon$ & $\varphi 36$ & $18 / \varepsilon \varepsilon, 9 / 2,8 / \varepsilon, 3 / \varepsilon$ \\
\hline
\end{tabular}

- For the tuple of states $(3,1,1), \varphi 16$

In our proposed method, we have selected a state in $A_{j}(j \neq 1)$ which ignores all the common inputs in $W_{1}^{\prime}$. So, each formula includes a state which returns the same response as the specification (e.g. $\varphi 11$ for the tuple of states $(0,1,1))$, and it may include a state whose response for a common input is $\varepsilon$ (e.g. $\varphi 12$ for the tuple of states $(0,1,1)$ ).

For the sub-IUT FSM2, we can get the following logical product of formulas using state variables in Table 8.

$$
(\varphi 21 \vee \varphi 22) \wedge \varphi 23 \wedge(\varphi 24 \vee \varphi 25) \wedge \varphi 26
$$

For the sub-IUT FSM3, we can get the following logical product of formulas using state variables in Table 9.

$$
(\varphi 31 \vee \varphi 32) \wedge \varphi 33 \wedge(\varphi 34 \vee \varphi 35) \wedge \varphi 36
$$

On all of the cases, the formula for each tuple of states always has one state whose response is expected on the specification, and it may have another state whose response is equal to the expected response except that the output for the common input is changed to $\varepsilon$. And also, the set of formulas for one sub-IUT has no same state variables, that is, all state variables in the set of formulas are different. Since we assume that the number of states of each sub-IUT $I_{i}$ does not exceed the number of states $N_{i}$ of the corresponding subspecification $A_{i}$, we must select at most $N_{i}$ state variables to be true in order to make all of $N_{i}$ formulas be true. So, we cannot select one state variable to be true so that two formulas can be true together. Then we must select only one of state variables in each formula to be true so that we make all of $N_{i}$ formulas be true.

If we select the state variable whose response is not the expected output $y$ but the empty output $\varepsilon$, we can consider that the output $y$, which we observed when we gave the characterization set to the IUT, was obtained from an other sub-IUT $I_{j}$. However, the formulas for the other sub-IUT $I_{j}$ have only state variables whose response is expected on the specification or equal to the expected response except that the output for the common input is changed to $\varepsilon$. So, each state in the other sub-IUT $I_{j}$ cannot produce the 
output $y$. Also, because of the limitation on the number of state variables to be true, we cannot select so much number of state variables.

From the above, all we can do is to select the state variables whose response is equal to the expected response on the specification in order to make all formulas be true. That is the reason why our method is correct for state identification.

We can identify the following four states for FSM1.

$$
\varphi 11, \varphi 13, \varphi 14, \varphi 16
$$

We can also identify all states in FSM2 and FSM3.

Here, we can say something about non-deterministic behavior of the IUT. Until now, when we give the IUT a transfer sequence $v$ which can make $A_{i}$ move to $s_{p}$, we cannot guarantee that the state of the sub-IUT $I_{i}$ is $s_{p}$ truly. However, now we can say that (1) each sub-IUT $I_{i}$ has a state corresponding to each state in the sub-specification $A_{i}$, and (2) we can exactly lead the state of a sub-IUT to any state by the transfer sequence used to identify the state.

\subsection{Correctness of Confirmation of Transitions}

From now, we explain why by our proposal method (by giving test suite $v . x . W_{i}$ or $\left.v . W \cup v . x . W_{i}^{\prime}\right)$, we can check whether each transition $s_{p} \stackrel{x / y}{\rightarrow} s_{q}(x \in$ $X, y \in Y \cup\{\varepsilon\})$ in the sub-specification $A_{i}$ is correctly implemented on the corresponding sub-IUT $I_{i}$.

(Case 1)

If the input $x$ is not a common input, we give the IUT the test suite $v \cdot x \cdot W_{i}^{\prime}$ where $v$ is the transfer sequence used to identify the state $s_{p}$. We can guarantee that the starting state of the transition $x / y$ is $s_{p}$ truly since $v$ is the sequence used to identify the state $s_{p}$. And by observing outputs from the IUT for $x . W_{i}^{\prime}$, we can identify the state after $x / y$ is executed.

(Case 2)

If the input $x$ is a common input, we give the IUT the test suite $v \cdot W \cup v \cdot x \cdot W_{i}^{\prime}$. In general, when testing of state identification has not been finished, each sub-IUT may have a wrong state whose response is not equal to that on the sub-specification even if the response of the sub-IUT for all of $W_{i}^{\prime}$ is correct. But if testing of state identification has been finished, and if we can get the expected response, it shows the existence of the state of $I_{i}$ corresponding to a state of the sub-specification $A_{i}$ (because there are not any other possibilities). Then if we can observe the expected outputs when we give $W$ for the tuple of states which the transfer sequence $v$ leads $\left(s s_{p}^{i}=\left(s_{1}^{i}, s_{2}^{i}, \cdots, s_{k}^{i}\right)\left(s_{i}^{i}=s_{p}\right)\right)$, we can guarantee that we have led the IUT to $s s_{p}^{i}$.

Next we explain why we can confirm transitions by the test suite $v \cdot x . W_{i}^{\prime}$ by dividing into three cases.

(Case 2.1) 
We first consider the case that the state is changed by execution of the transition $s_{p} \stackrel{x / y}{\rightarrow} s_{q}(x \in X, y \in Y \cup\{\varepsilon\})$. Because we have constructed the tuple of states so that a state $s_{j}^{i}$ can ignore the common input $x$, the output must be only $y$ when the input $x$ is given at $s s_{p}^{i}$. At this time, by observing the response for $W_{i}^{\prime}$, we can check whether the common input $x$ is taken into other DFSMs. If another DFSM takes the input $x$ and produces the output $y$, and if the state $s_{p}$ of the sub-IUT $I_{i}$ is badly implemented as it can ignore the input $x$, then the response for $W_{i}^{\prime}$ is not for $s_{q}$ but $s_{p}$, and then we can find the error.

(Case 2.2)

Secondly we consider the case that the state is not changed by execution of the transition $s_{p} \stackrel{x / y}{\rightarrow} s_{q}(x \in X, y \in Y \cup\{\varepsilon\})$, and the output $y$ is $\varepsilon$. Since we have constructed the tuple of states $s s_{p}^{i}$ so that every state in $s s_{p}^{i}$ ignores the common input $x$, we can find the error if we cannot observe the output $\varepsilon$ after giving $x$. We can also find the error that $s_{p}$ and $s_{q}$ are not the same if the response for $W_{i}^{\prime}$ is different from $s_{p}$ 's response.

(Case 2.3)

Lastly, we consider the case that the state is not changed by execution of the transition $s_{p} \stackrel{x / y}{\rightarrow} s_{q}(x \in X, y \in Y \cup\{\varepsilon\})$, and the output $y$ is not $\varepsilon$. On this case we can test in a similar way. We have constructed the tuple of states $s s_{p}^{i}$ so that every state in $I_{j}(j \neq i)$ ignores the common input $x$. On this case that the output $y$ is not $\varepsilon$, however, we should consider that the IUT may be implemented as that state $s_{p}$ of $I_{i}$ can ignore the common input $x$ and a state of another sub-IUT $I_{j}$ can give the output $y$ without changing its state. On the condition of $\left(s_{p}=s_{q}\right)$ and $(y \neq \varepsilon)$, we cannot deny such a possibility only by confirmation of the transitions of $I_{i}$. However the possible error is that the output $y$ may be changed to $\varepsilon$ like the testing for state identification. We can guarantee that there is not such a possibility by confirmation of all transitions in each sub-IUT $I_{j}$. That is, by confirming of the transitions at all states of each sub-IUT $I_{j}$, we can assure that each sub-IUT $I_{j}$ can give either an output $z$ which can be used only by $A_{j}$ or only $\varepsilon$. So we can also assure that $I_{j}$ cannot give the output $y$ which can be used only by $A_{i}$, and finally we can guarantee that $I_{i}$ gives the output $y$ truly.

From the above, our proposal method guarantees that the implementation of transitions is correct.

\section{EXAMPLE}

We have applied our testing method to the abracadabra protocol. We have simplified an Estelle specification of the abracadabra protocol in [3] and modified it so that three sending processes run in parallel. Fig. 3 is its specification in our model where $\mathrm{I} / \mathrm{O}$ symbols are represented symbolically. From this spec- 


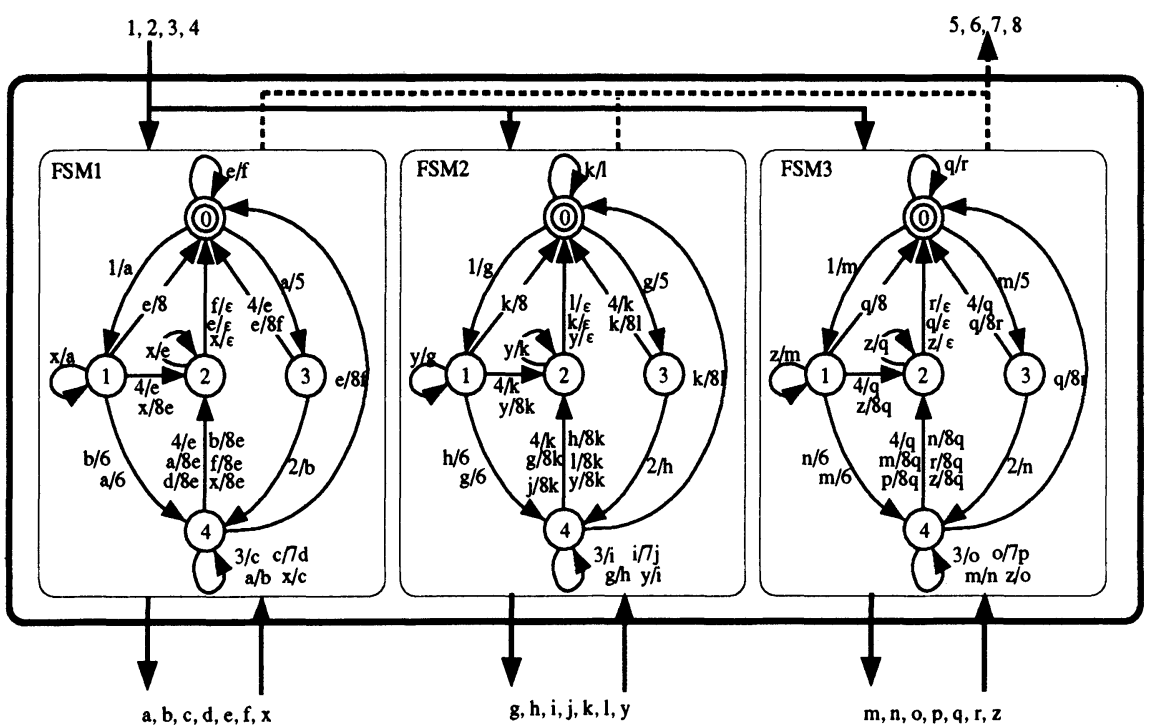

Figure 3 Example Spec. for Abracadabra Protocol.

ification, we have constructed characterization sets satisfying the conditions mentioned in Section 4.1.

\section{CONCLUSION}

In this paper, we propose a conformance testing method for communication protocols modeled as a set of DFSMs with common inputs. In the proposed method, we check each DFSM independently. So, the cost is only proportional to the sum of the numbers of states and transitions of DFSMs. Although we assume the existence of characterization sets which satisfy some conditions, we believe many communication protocols satisfy the conditions.

One of the future work is to extend the class of DFSMs so that we can treat the communications between DFSMs and internal actions in DFSMs.

\section{REFERENCES}

[1] T. S. Chow : "Testing Software Design Modeled by Finite-State Machines", IEEE Trans. on Software Engineering, Vol. 4, No. 3, pp.178-186, 1978.

[2] G. Gonenc: "A Method for the Design of Fault-Detection Experiments", IEEE Trans. on Computers, Vol. C-19, No. 6, pp. 551-558, 1970.

[3] ISO : "Information Technology - Open Systems Interconnection - Guidelines for the application of Estelle, LOTOS and SDL", ISO/IEC/TR $10167,1991$. 
[4] H. Kloosterman : "Test Derivation from Nondeterministic Finite State Machines", Proceedings of the IFIP TC6 Fifth International Workshop on Protocol Test Systems, pp.297-308, 1992.

[5] D. Lee, K. K. Sabnani, D. M. Kristol and S. Paul : "Conformance Testing of Protocols Specified as Communicating FSMs", Proceedings of IEEE INFOCOM'93, pp.115-127, 1993.

[6] G. Luo, G. v. Bochmann and A. Petrenko : "Test Selection Based on Communicating Nondeterministic Finite State Machines using a Generalized Wp-method", IEEE Trans. on Software Engineering, Vol. 20, No. 2, pp.149-162, 1994.

[7] S. Naito and M. Tsunoyama : "Fault Detection for Sequential Machines by Transition Tours", Proceedings of the 11th IEEE International Symposium on Fault Tolerant Computing, pp.238-243, 1981.

[8] D. P. Sidhu and T.-K. Leung : "Formal Methods for Protocol Testing : A Detailed Study", IEEE Trans. on Software Engineering, Vol. 15, No. 4, pp.413-426, 1989.

[9] K. K. Sabnani and A. T. Dahbura : "A Protocol Testing Procedure", Computer Networks and ISDN Systems, Vol. 15, No. 4, pp.285-297, 1988.

[10] P. H. Starke : "Abstract Automata", North-Holland/American Elsevier, 1972.

[11] P. Tripathy and K. Naik : "Generation of Adaptive Test Cases from Nondeterministic Finite State Models", Proceedings of the IFIP TC6 Fifth International Workshop on Protocol Test Systems, pp.309-320, 1992.

[12] A. Ulrich and S. T. Chanson : "An Approach to Testing Distributed Software Systems", Proceedings of the IFIP WG6.1 15th International Symposium on Protocol Specification, Testing and Verification, pp.121-136, 1995.

[13] T. Kaji, T. Higashino and K. Taniguchi : "A Conformance Testing for Communication Protocols Modeled as Coupled DFSMs", Information Processing Society of Japan (IPSJ) SIG Notes 96-DPS-74, Vol. 96, No. 12, pp.19-24, 1996 (in Japanese).

\section{BIOGRAPHY}

Atsushi Fukada is a Master student of the Department of Information and Computer Sciences, Osaka University, Japan. His research interests include testing of communication protocols. Tadashi Kaji finished his Master course at the same department in 1996 and is currently working in Hitachi Co. Ltd.

Teruo Higashino and Kenichi Taniguchi are an Associate Professor and a Professor in the same department, respectively. Masaaki Mori is a Professor in Shiga University, Japan. Their research interests include communication protocols, formal description techniques and software engineering. 